\title{
\begin{tabular}{l|l} 
Mibraries & DSpace@MIT
\end{tabular}
}

\author{
MIT Open Access Articles
}

\section{Exo-C: a probe-scale space observatory for direct imaging and spectroscopy of extrasolar planetary systems}

The MIT Faculty has made this article openly available. Please share how this access benefits you. Your story matters.

Citation: Stapelfeldt, Karl R., et al. "Exo-C: A Probe-Scale Space Observatory for Direct Imaging and Spectroscopy of Extrasolar Planetary Systems." Proceedings Volume 9605, Techniques and Instrumentation for Detection of Exoplanets VII, 9-13 August, 2015, San Diego, California, edited by Stuart Shaklan, 2015, p. 96050T. (c) 2015 SPIE.

As Published: http://dx.doi.org/10.1117/12.2191720

Publisher: SPIE

Persistent URL: http://hdl.handle.net/1721.1/115191

Version: Final published version: final published article, as it appeared in a journal, conference proceedings, or other formally published context

Terms of Use: Article is made available in accordance with the publisher's policy and may be subject to US copyright law. Please refer to the publisher's site for terms of use. 


\section{Exo-C: a probe-scale space observatory for direct imaging and spectroscopy of extrasolar planetary systems}

Karl R. Stapelfeldt, Frank G. Dekens, Michael P. Brenner, Keith R. Warfield, Ruslan Belikov, et al.

Karl R. Stapelfeldt, Frank G. Dekens, Michael P. Brenner, Keith R. Warfield, Ruslan Belikov, Paul B. Brugarolas, Geoffrey Bryden, Kerri L. Cahoy, Supriya Chakrabarti, Serge Dubovitsky, Robert T. Effinger, Brian Hirsch, Andrew Kissil, John E. Krist, Jared J. Lang, Mark S. Marley, Michael W. McElwain, Victoria S. Meadows, Joel Nissen, Jeffrey M. Oseas, Chris Pong, Eugene Serabyn, Eric Sunada, John T. Trauger, Stephen C. Unwin, "Exo-C: a probe-scale space observatory for direct imaging and spectroscopy of extrasolar planetary systems," Proc. SPIE 9605, Techniques and Instrumentation for Detection of Exoplanets VII, 96050T (16 September 2015); doi: 10.1117/12.2191720

Event: SPIE Optical Engineering + Applications, 2015, San Diego, California, United States 


\title{
Exo-C: A probe-scale space observatory for direct imaging and spectroscopy of extrasolar planetary systems
}

\author{
Karl R. Stapelfeldt ${ }^{a}$, Frank G. Dekens ${ }^{b}$, Michael P. Brenner ${ }^{b}$, Keith R. Warfield ${ }^{b}$, \\ Ruslan Belikov $^{c}$, Paul B. Brugarolas ${ }^{b}$, Geoffrey Bryden ${ }^{b}$, Kerri L. Cahoy ${ }^{d}$, \\ Supriya Chakrabarti ${ }^{e}$, Serge Dubovitsky ${ }^{b}$, Robert T. Effinger ${ }^{b},{\text { Brian } \text { Hirsch }^{b} \text {, Andrew Kissil }}^{b}$, \\ John E. Krist ${ }^{b}$, Jared J. Lang ${ }^{b}$, Mark S. Marley ${ }^{c}$, Michael W. McElwain ${ }^{a}$, \\ Victoria S. Meadows ${ }^{f}$, Joel Nissen $^{b}$, Jeffrey M. Oseas ${ }^{b}$, Chris Pong $^{b}$, \\ Eugene Serabyn ${ }^{b}$, Eric Sunada ${ }^{b}$, John T. Trauger ${ }^{b}$, Stephen C. Unwin ${ }^{b}$ \\ ${ }^{a}$ NASA Goddard Space Flight Center, Code 667, Greenbelt MD 20771 USA \\ ${ }^{b}$ Jet Propulsion Laboratory, California Institute of Technology, Pasadena CA 91109 USA \\ ${ }^{c}$ NASA Ames Research Center, Moffet Field CA 94035 USA \\ ${ }^{d}$ Massachusetts Institute of Technology, Cambridge MA 02139 USA \\ ${ }^{e}$ Univ. of Massachusetts, Lowell MA 01854 USA \\ fUniv. of Washington, Seattle WA 98195 USA
}

\begin{abstract}
"Exo-C" is NASAs first community study of a modest aperture space telescope mission that is optimized for high contrast observations of exoplanetary systems. The mission will be capable of taking optical spectra of nearby exoplanets in reflected light, discovering previously undetected planets, and imaging structure in a large sample of circumstellar disks. It will obtain unique science results on planets down to super-Earth sizes and serve as a technology pathfinder toward an eventual flagship-class mission to find and characterize habitable Earth-like exoplanets. We present the mission/payload design and highlight steps to reduce mission cost/risk relative to previous mission concepts. Key elements are an unobscured telescope aperture, an internal coronagraph with deformable mirrors for precise wavefront control, and an orbit and observatory design chosen for high thermal stability. Exo-C has a similar telescope aperture, orbit, lifetime, and spacecraft bus requirements to the highly successful Kepler mission (which is our cost reference). Much of the needed technology development is being pursued under the WFIRST coronagraph study and would support a mission start in 2017, should NASA decide to proceed. This paper summarizes the study final report completed in March 2015.
\end{abstract}

Keywords: Exoplanets, high contrast imaging, optical astronomy, space mission concepts

\section{INTRODUCTION}

Over the past five decades, NASA has carried out ambitious space observatory projects designed to study the universe at new wavelengths with improved spatial resolution, spectral resolution, and field of view - and with precise timing or photometry. In the 21st century, exoplanet research has emerged as a new focus for astrophysics and offers new space mission opportunities to explore. A new observational domain - imaging at very high contrasts and very small angular separations - must be opened if we are to understand the properties, formation, and evolution of planetary systems around stars like the Sun. The Exo-C probe mission study is an effort chartered by NASA HQ in 2013, with the goal of designing a modest-sized space observatory designed from the outset to meet the requirements of high contrast imaging, and to do so with a $\$ 1$ billion cost cap. It brings together a $1.4 \mathrm{~m}$ aperture, active wavefront control, internal coronagraphs with significant heritage in laboratory demonstrations, and a highly stable spacecraft environment to enable optical wavelength studies of nearby planetary systems at billion- to-one contrast. Exo-C will directly image and take spectra of planets beyond the reach of other telescopes. The mission and hardware design is patterned on the highly successful Kepler mission to achieve the same goal of delivering groundbreaking exoplanet science at an affordable cost.

Send correspondence to karl.r.stapelfeldt@nasa.gov, Telephone: (301) 2863328

Techniques and Instrumentation for Detection of Exoplanets VII, edited by Stuart Shaklan, Proc. of SPIE Vol. 9605 96050T · (c) 2015 SPIE · CCC code: 0277-786X/15/\$18 · doi: 10.1117/12.2191720 

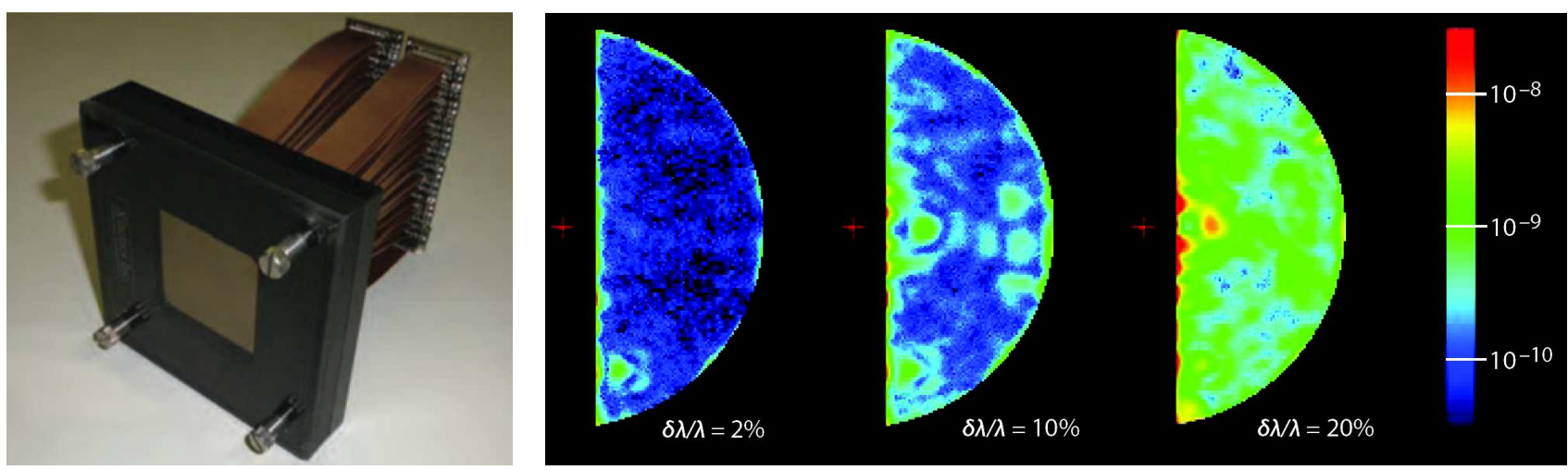

Figure 1. Exo-C enabling technology. Left: A Xinetics precision deformable mirror in 48x48 format. Right: High contrast dark fields created using this deformable mirror with a hybrid Lyot coronagraph in a vaccum testbed at the Jet Propulsion Laboratory. The occulted light source is located at the position of the red cross; the color scale indicates image contrast.

The key enabling technology for Exo-C is a precision deformable mirror (DM) capable of being commanded and maintained at sub-angstrom accuracy. In conjunction with additional coronagraph elements to suppress diffraction, the DM is used to clear a high-contrast dark hole around the target star out to a maximum radius of $N \lambda / 2 D$, where $\mathrm{N}$ is the linear $\mathrm{DM}$ actuator count, $\lambda$ is the wavelength, and $D$ is the telescope aperture diameter. Laboratory demontrations to date show that the needed level of $10^{-9}$ contrast can now be achieved for unobscured pupils in a static system with optical bandwidths up to 20\% (Figure 1). Past exoplanet direct imaging mission concept studies utilizing this approach include ACCESS, EPIC, and PECO (Trauger et al. 2010; Clampin et al. 2010; Guyon et al. 2010). Exo-C brought previously competing groups together in a single Science and Technology Definition Team supported by an Engineering Design Team at NASA/JPL. Technical readiness by 2017 and launch readiness in 2024 were required for the mission concept.

\section{SCIENCE GOALS \& REQUIREMENTS}

Exo-C's prime science targets are planetary systems within $20 \mathrm{pc}$ of the sun. By the year Exo-C would launch, preceding ground and space telescopes will have identified stars hosting short-period transiting planets and gas giant planets on orbits $\sim<5 \mathrm{AU}$. The atmospheric properties of hot, close-in planets will have been probed in the near-infrared by transit spectroscopy; and for hot, young planets by near-infrared adaptive optics imaging. While these advances will be remarkable scientific milestones, they will fall well short of the goal of obtaining images and spectra of planetary systems like our own. Exo-C would study cool planets in reflected light at visible wavelengths, ranging from gas giants down to super Earths, at separations from 1-9 AU, around nearby stars like the Sun. The mission design parameters needed to achieve this science goal are listed below.

\begin{tabular}{|lc|}
\hline Primary mirror diameter & $1.4 \mathrm{~m}$ \\
Raw speckle contrast & $10^{-9}$ at the IWA \\
Contrast stability after control & $10^{-10}$ or better at the IWA \\
Spectral coverage & $450-1000 \mathrm{~nm}$ \\
Inner working angle (IWA) & $2 \lambda / D=0.16^{\prime \prime} @ 550 \mathrm{~nm}$ \\
Outer working angle (OWA) & $>20 \lambda / D=2.6^{\prime \prime} @ 800 \mathrm{~nm}$ \\
Binary spillover light & $3 \times 10^{-8}$ contrast @ $8^{\prime \prime}$ \\
Spectral resolution $\lambda>500 \mathrm{~nm}$ & $\mathrm{R}=70$ \\
Astrometric precision & $<30$ milliarcsec \\
Imaging camera field of view & $42^{\prime \prime}$ \\
Imaging Spectrograph field of view & $2.2^{\prime \prime}$ \\
Mission lifetime & 3 years \\
\hline
\end{tabular}



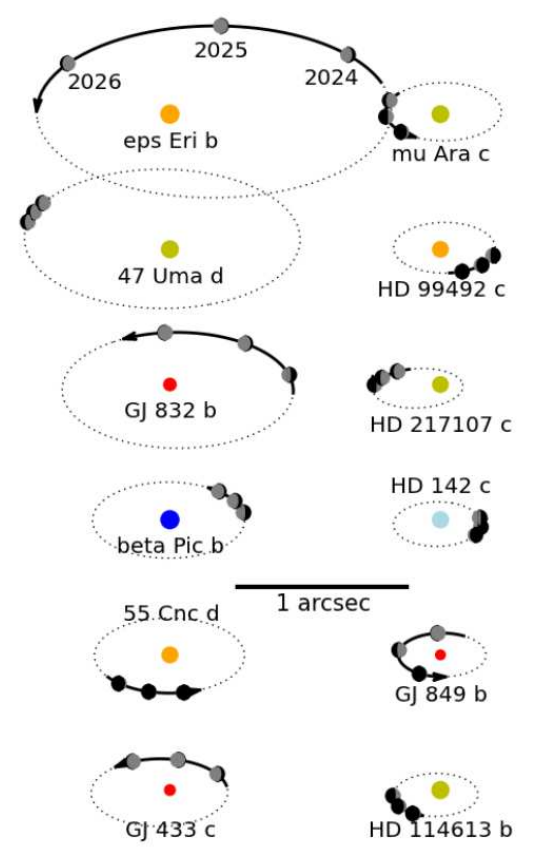

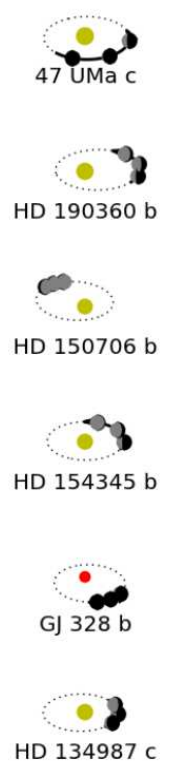

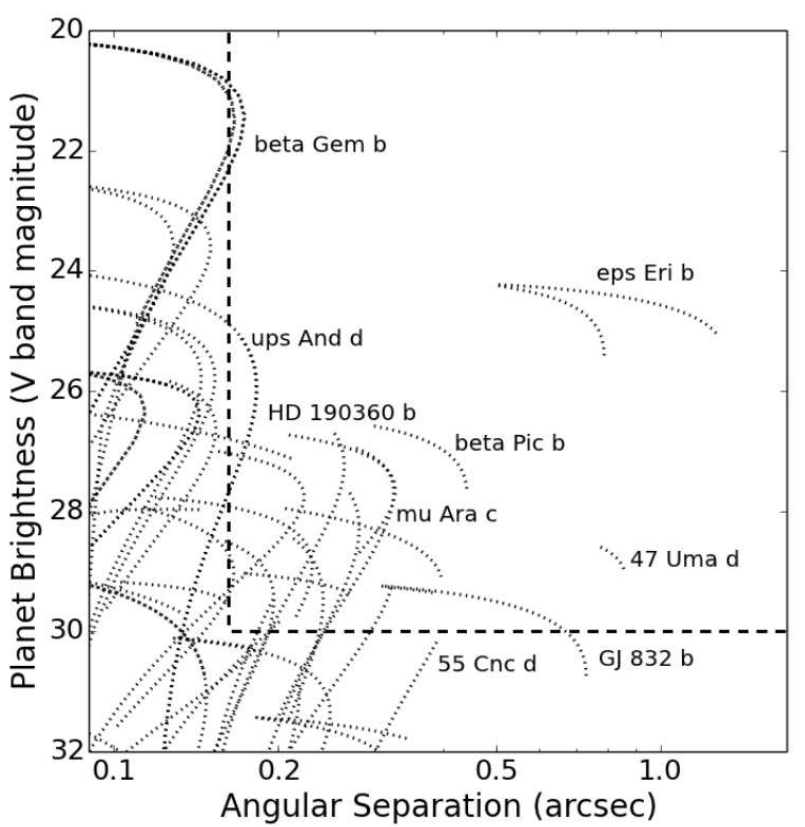

Figure 2. Known Planetary Targets. Exo-C will observe a sample known exoplanets whose orbits have already been constrained by groundbased measurements. For an assumed orbital inclination of $70^{\circ}$, the illumination of the widestseparation/brightest planets is shown for three epochs from 2024 to 2026 (left panel). The brightness of each planet is shown as a function of orbital separation over the same time period (right panel). Targets must have sufficient angular separation $\left(\geq 0.16^{\prime \prime}\right)$ and must be bright enough (visual magnitude $<30$ ) to be detected.

\subsection{Exoplanet Spectra}

Radial velocity (RV) surveys have detected many exoplanets around nearby stars, many of which are prime targets for Exo-C imaging. Beyond simply knowing that a planet is present, RV detections also constrain the orbital separation and relative illumination as a function of time, such that an optimal epoch for observation can be chosen within the Exo-C mission lifetime. RV measurements by themselves determine the product of the planet mass and the sine of the orbital inclination. Imaging detections of a RV planet provide astrometry which resolves the $\sin (i)$ ambiguity and thus specifies the planet mass, which then aids in subsequent interpretation of its atmospheric spectrum. The RV planets orbit mature, quiet stars for which excellent elemental abundances can be derived. This will allow meaningful comparison of abundances measured in the planetary atmospheres to those of the star. As seen in Figure 2, about a dozen known RV planets have large enough angular separation and are bright enough for Exo-C to image. With the instrument inner working angle (IWA) increasing with wavelength, a full spectrum from $0.5-1.0 \mu \mathrm{m}$ can be obtained for about half of these planets.

The spectral range 450-1000 $\mathrm{nm}$ encompasses many molecular absorption bands of varying strengths of methane, water, and ammonia (Figure 3). The long wavelength cutoff is chosen to allow some detection of continuum on the red side of the $940 \mathrm{~nm}$ water band and the short wavelength cutoff is motivated by the relatively bland Rayleigh and haze-scattering spectrum expected in the blue for giant planets.

For spectral characterization, a spectral resolution of $\mathrm{R}=70$ was chosen as the minimum required to detect and characterize methane bands with a variety of strengths, as well as the water band at $940 \mathrm{~nm}$ for Jovian planets. Additionally, $\mathrm{R}=70$ is optimal for detecting the $\mathrm{O}_{2}$ A-band at $760 \mathrm{~nm}$, a potential biosignature, should a super-Earth planet be found in the habitable zone of one of the stars in the $\alpha$ Centauri system. A signal to noise of 10 would be sufficient for the measurement of these features. For planets too faint for full spectroscopy, color filter imaging measurements will be made instead. 

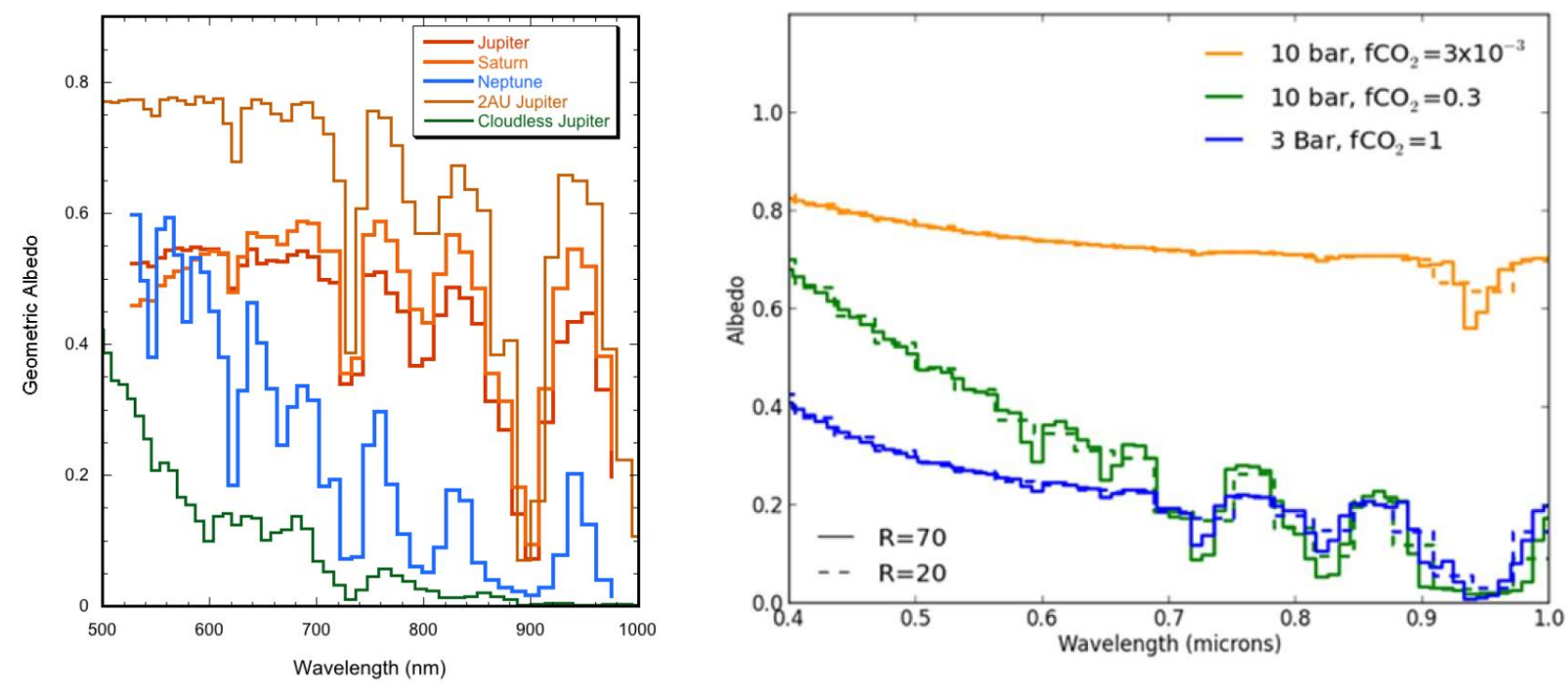

Figure 3. Example Target Spectra. Left: Geometric albedo spectra of real and model giant planets convolved to R = 70 spectral resolution. Shown are Jupiter, Saturn, and Neptune (Karkoschka et al. 2011), along with a warm Jupiter and a cloudless Jupiter three times enhanced in heavy elements from (Cahoy) et al. 2010. The warm Jupiter is very bright, while conversely the cloudless Jupiter is extremely dark. Right: Simulated spectra of super-Earth atmospheres with different total pressures and amounts of $\mathrm{CO}_{2}$ and water vapor.

\subsection{Exoplanet Discovery Surveys}

RV surveys are incomplete for orbital periods $>12$ years, for hotter stars lacking strong metallic lines in their spectra, for stars with strong spot and flare activity, and for planets in nearly face-on orbits. Multi-epoch imaging with Exo-C has the potential to discover planets beyond RV limits around as many as 150 nearby stars (Figure 4). There are more than 70 stars within 25 pc that host close-in RV planets and would be prime targets for outer planet searches. Two upcoming missions to detect transiting planets, TESS in 2017 and PLATO in 2024, will identify additional nearby stars with planetary systems; and the European Space Agency's Gaia mission will identify additional new gaint planets using precision astrometry. The number of known, nearby planetary systems can thus be expected to grow significantly between now and Exo-C's 2024 launch.

Exo-C's contrast capability will permit detections of Jupiter-like planets on orbits out to 9 AU, Neptune-like planets out to $3 \mathrm{AU}, 3 \mathrm{R}_{\oplus}$ mini-Neptunes out to $2 \mathrm{AU}$, and $1.5 \mathrm{R}_{\oplus}$ super-Earths at $1 \mathrm{AU}$. The optimal stars for imaging of exoplanets in reflected light are both bright and near the Sun. They are generally hotter than 6500 $\mathrm{K}$, making them a substantially different stellar population from the sample monitored with the radial velocity technique by groundbased telescopes (Howard and Fulton 2015). The optimal stars are also 2 magnitudes brighter than the RV planet host stars known today, with the important implication that the brightest exoplanets that yield the best spectra will be the exoplanets discovered by Exo-C itself.

Particularly important targets will be the two Sun-like stars of the $\alpha$ Centauri binary system, the Sun's nearest neighbor. Because this target is so close, its habitable zone is easily observed and an Earth analog would be as bright as visual magnitude 26. The spillover light from the companion star (appearing at $8^{\prime \prime}$ separation in 2024) makes imaging detection of such a planet difficult. It is neverthless possible here and in a handful of other nearby stars, as long as Exo-C achieved excellent contrast stability.

\subsection{Disk Imaging}

Debris disks trace the dust liberated by ongoing collisions in belts of asteroidal and cometary parent bodies. In addition to revealing the location of these belts, debris dust serves as a tracer of the dynamical signature of unseen planets. Exo-C will be capable of resolving substructures in these dust disks such as rings, gaps, warps, 


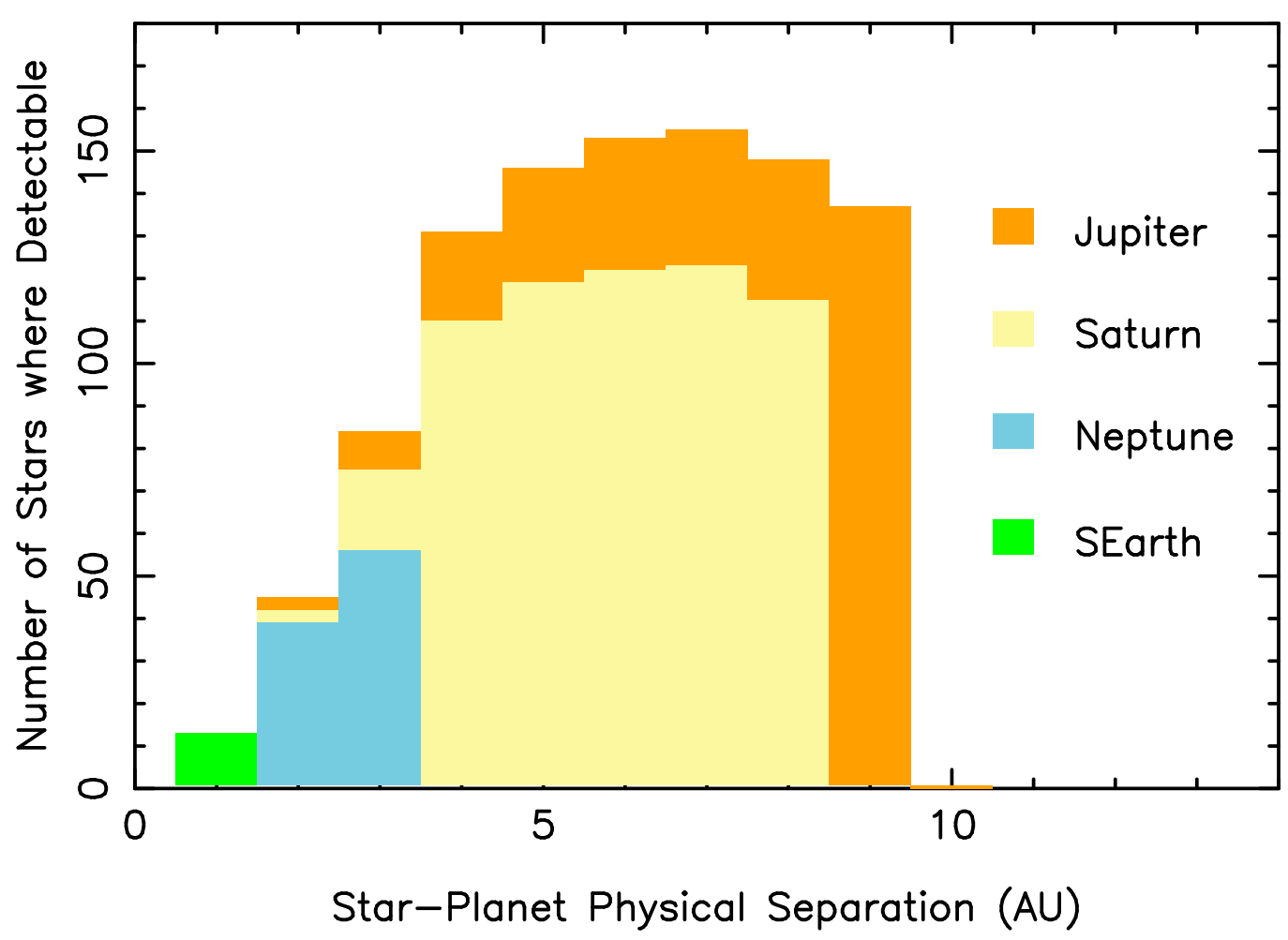

Figure 4. Exo-C planet search space. These four histograms show the number of nearby Hipparcos stars where planets of various sizes and orbital radii can be detected in two visits and within a cutoff integration time. Due to the $1 / \mathrm{r}^{2}$ law, smaller planets must be located closer to the star to be detected at the same fiducial contrast level of $10^{-9}$. The left side of these distributions is largely defined by Exo-C's inner working angle, while the right side is defined by a limiting contrast of $3 \times 10^{-10}$ derived from telescope stability considerations.

and asymmetries driven by planetary perturbations. With contrast improved $1000 \times$ over the Hubble Space Telescope (HST), Exo-C will be sensitive enough to detect disks as tenuous as our own Kuiper Belt, enabling comparative studies of dust inventory and properties across a range of stellar ages and spectral types. Several hundred debris disk targets will be surveyed, including 1) nearby stars with far-infrared excess detected by the Spitzer, Herschel, and WISE missions; and 2) RV planet systems where sculpted dust features might be seen. A small survey of protoplanetary disks will reveal how small dust particles are distributed with respect to the larger particles mapped in images from the Atacama Large Millimeter/submillimeter Array (ALMA).

Exo-Cs inner working angle of $0.16^{\prime \prime}$ at $550 \mathrm{~nm}$ is sufficient to spatially resolve the habitable zones of $\sim 25$ Sun-like stars and another $\sim 75$ stars with earlier spectral types. A survey of these targets will search for extended surface brightness from exozodiacal dust, to limits within a few times the dust levels found in our own Solar System. The detected surface brightness will constrain the dust inventory and albedo, thus helping to define the background light levels against which future missions will observe Earth-like extrasolar planets. In the nearest examples, Exo-C images may be able to indirectly detect habitable planets from asymmetric structures they induce in the habitable zone dust distribution.

\subsection{Other Astrophysics and Planetary Science}

Exo-C's imaging camera will carry a small filter set that will enable optical imaging and photometry of any bright (visual magnitude $<13$ ) astronomical target over its modest field of view. This could include temporal studies of solar system objects, if the pointing system was enhanced to track moving targets. The coronagraph could be utilized to study dust shells around post-main sequence objects and the host galaxies of quasars or active galactic nuclei. Imaging of fields without a bright guidestar would require a major redesign of the pointing 
architecture. Exo-C's optical bench has sufficient volume to accommodate a second instrument and the payload mass budget would also allow this. A near-IR spectrograph for transit spectroscopy would be one option to consider. The costs of a second instrument would require additional funds above the the mission's $\$ 1 \mathrm{~B}$ cost cap.

\section{ARCHITECTURE TRADES}

\subsection{Telescope Type and Aperture}

From a performance perspective, the use of an unobscured telescope form is preferred for coronagraphy. This choice maximizes the system throughput and image core flux, directly leading to more favorable integration times and a larger science program. Five other factors (polarization influence, fabrication complexity, structural considerations, optical design complexity, and binary target performance) yielded no net distinction or only very weakly favored one form over the other. An f/2.5 primary was chosen in order to preserve the integrity of the high-contrast wavefront correction in both polarizations simultaneously.

The science performance clearly increases with aperture size, since it increases the light collected. More important, the clear aperture size for a coronagraph sets the inner working angle (IWA). This in turn directly affects the number of known radial velocity (RV) exoplanets for which Exo-C is able to obtain spectra, and the number of Super-Earths that Exo-C would be capable of detecting. For the known RV planets shown in Fig. 2, a $1.5 \mathrm{~m}$ aperture provides sufficient IWA to make spectra of 10 targets out to $\lambda=800 \mathrm{~nm}$ and could image $2 \mathrm{R}_{e}$ super-Earth targets if they are present around 10 other stars near the sun. A $1.4 \mathrm{~m}$ aperture provides almost the same performance, while a noticable degradation is seen for a $1.3 \mathrm{~m}$ aperture. All three aperture sizes appeared feasible from a mass perspective for our assumed intermediate class launch vehicle, while cost considerations set $1.5 \mathrm{~m}$ as the maximum size likely to be afforded given the $\$ 1 \mathrm{~B}$ cost cap. To balance cost and science, a $1.4 \mathrm{~m}$ aperture was selected in fall 2014 as the final design point for the Exo-C study.

\subsection{Orbit and Telescope Stability}

Thermal stability of the telescope is a prime consideration for achieving the stable optical wavefronts needed for $10^{-9}$ contrast imaging. Earth orbits (LEO, sun-synchronous, GEO) were not considered because the varying radiative inputs on the telescope could require complex countermeasures to control. L2 and Earth-trailing orbits provide the most benign thermal environments. These two options were compared in the areas of science capability (a function of sky accessibility and target availability) and a model-based cost estimation to determine engineering and operation cost differences to access and maintain the two orbits. Initial examination of this trade showed no significant target availability or data return advantage for Exo-C in L2 orbit over an Earth-trailing spacecraft. As a result, the major driver for orbit selection was the overall mission cost. Due to the need to carry extra propulsion capability for orbit maintenance and the need for a navigation team to execute that maintenance, L2 orbit would entail additional costs that the Earth-trailing orbit would not. Therefore, the Earth-trailing orbit was selected as the baseline orbit for the Exo-C Probe study.

In addition to choosing a stable orbit, body-fixed solar arrays and high-gain antenna were selected over articulated ones. This results in a stiffer observatory that will be less susceptable to dynamic excitation during slews or reaction wheel desaturations, and thus better pointing stability should be achieved. These choices are the same as those made by the Kepler mission to achieve their reknowned photometric stability.

\subsection{Science Instrument}

There are three areas where options were considered: science image path, fine guidance sensor path, and spectrometer path. It is desirable to maximize the number of common elements between these three optical paths so that a common wavefront control system can be used for all three. An important constraint is that spectral coverage must be obtained piecewise in $20 \%$ wide bands, as this is the current state of the art for high contrast wavefront control. Doing this this simultaneously in adjacent wavelength channels would require an excessively complex instrument - essentially four separate coronagraphs with their own pairs of deformable mirrors.

A single-path instrument with selectable elements (to provide coverage over the full waveband) was baselined. The fine guidance sensor (FGS) sees the light of the bright target star reflected off the coronagraphic mask and senses the pointing jitter at high rates. This signal can then be used to drive a fine-steering mirror (FSM) that 
keeps the star centered on the coronagraphic mask with no loss of signal to the science path. Broadband imaging is carried out with the science camera with different DM settings for each band. Spectroscopy is carried out with an integral field spectrometer (IFS) that shares the science field of view with the imaging camera, with a flip mirror to switch the input beam between the two. Implementation of an IFS has significant mission benefits. Its detector could perform as a reduced-capacity backup in the event of a failure of the imaging detector. It provides spectral diversity information of the residual speckle pattern, which will facilitate the derivation of wavefront control solutions, post-processing of the spectral images isolate planetary spectra from speckles, and the simultaneous spectroscopy of multiple planets and dust structures in the exoplanetary system.

Two basic configurations were examined to accommodate the coronagraph instrument. A lateral configuration, which places the instrument parallel and offset to the telescope axis, was selected for its ability to fulfill all desired functions while providing for best overall performance with a minimum total count and lowest angles of incidence on critical optical surfaces. This design is unique relative to the Hubble Space Telescope instruments, all of which were mounted behind the on-axis Cassegrain mirror. In a modest-size telescope like Exo-C, the lateral instrument bench provides more available room and better isolates the instrument from variable solar flux inputs during telescope pitch maneuvers. The lateral bench also allows the instrument design to avoid a high incidence angle reflections in the optical path from the telescope to the instrument. This is valuable because it preserves the integrity of the high contrast wavefront correction in both linear polarization states.

\subsection{Coronagraph Type}

Five coronagraph architectures were considered: Hybrid Lyot Coronagraph (HLC; Trauger et al. 2012), PhaseInduced Amplitude Apodization (PIAA; Guyon et al. 2012), Shaped Pupils (Carlotti et al. 2012), Vector Vortex (Mawet et al. 2012), and the Visible Nuller (Lyon et al. 2012). Following the process used in the Fall 2013 AFTA Coronagraph Working Group, optical performance simulations were developed with design inputs from the architecture advocates, and implemented in a common software environment. These simulations included the effects of pointing jitter which degrades contrast performance and the inner working angle. The simulations were input to codes that calculated integration times for spectroscopy of known RV planets through each coronagraph.

The results showed that (within the uncertainties) four of the five coronagraph types would produce similar science yields in the Exo-C observatory. The Shaped Pupil, by virtue of its larger inner working angle of $3 \lambda / D$, produced a significantly lower science yield and was thus elimated from consideration for Exo-C. Following the Fall 2013 AFTA Coronagraph working group evaluations, the Visible Nuller was judged to be too technically immature for readiness in 2017 and was also dropped. For the remaining three types, a second design iteration took place in the summer of 2014. The Hybrid Lyot coronagraph was chosen as the baseline for Exo-C on the basis of its higher level of technical readiness, as shown by its bandwidth and contrast performance in the laboratory (Lawson et al. 2013). The Vector Vortex and PIAA have the potential for better inner working angles, which would be very useful if the telescope proved to be stable enough. The final Exo-C PIAA design specifies a $2 \lambda / D$ IWA. This is less aggressive than than the design proposed for PECO (Guyon et al. 2010), thereby relaxing the telescope stability requirements and the mirror manufacturing tolerances that have limited performance in testbed demonstrations, and still providing $2.8 \times$ better throughput than the baseline HLC. If verified in the laboratory, the Exo-C PIAA design would offer greater science return in spectroscopy and planet search surveys. Both the Vector Vortex and Exo-C PIAA coronagraph types should continue their technical development, toward the possibility of becoming the baseline coronagraph for a later project start.

\section{BASELINE DESIGN}

\subsection{Payload Mechanical}

Exo-C has gravitated toward a Kepler-like design due to similar stability, aperture, and mission life requirements. With a total mission cost around $\$ 750 \mathrm{M}$ FY15 - well below the Probe study $\$ 1 \mathrm{~B}$ requirement - Kepler made an excellent starting point for the Exo-C design. Aside from the payload, Exo-C is very similar to Kepler in design, only needing to add a two- stage passive vibration isolation system to the original Kepler architecture. These passive isolators are flight proven technology. The only other planned changes to the bus are more reliable reaction wheels and some structural panel resizing. 


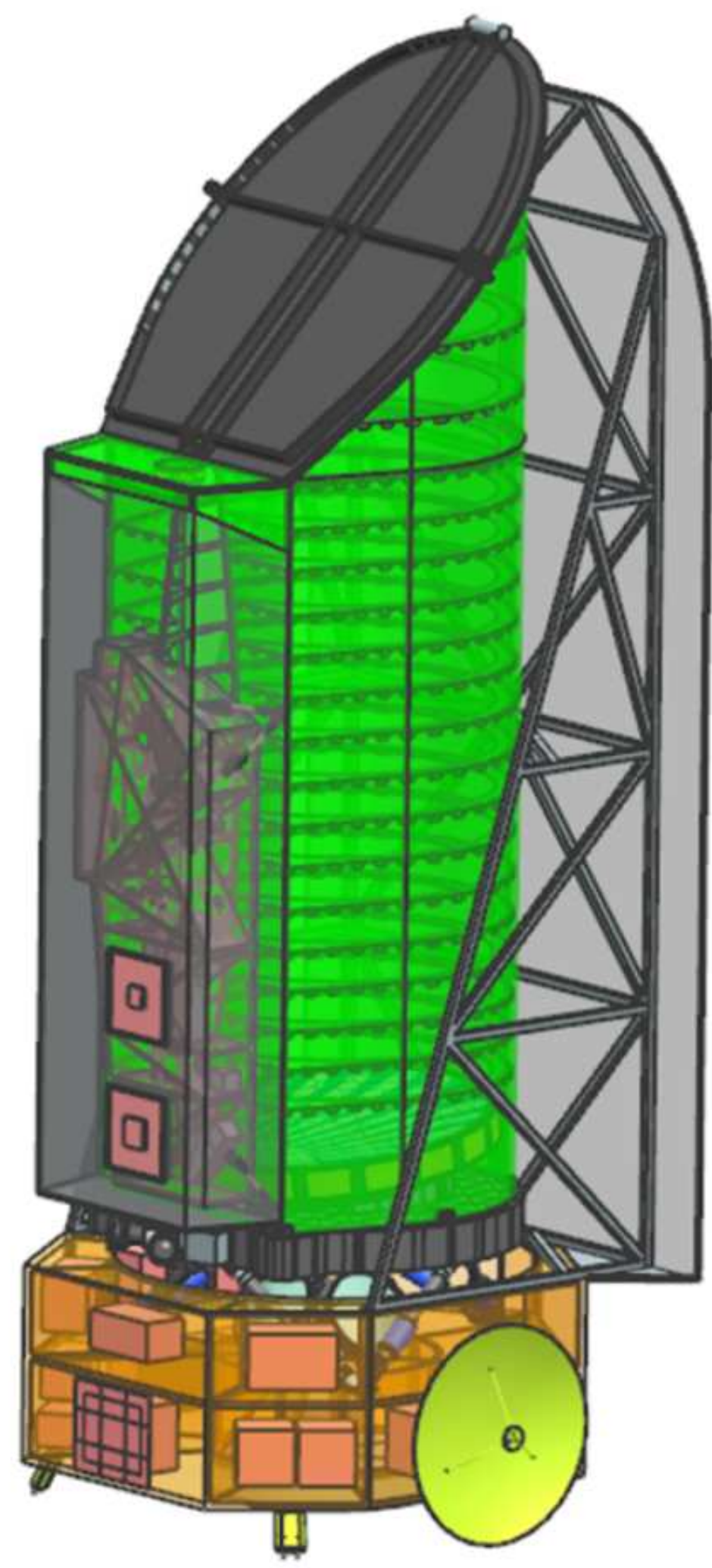

Figure 5. Final design configuration of the Exo-C observatory 
Exo-C consists of the instrument payload attached to the spacecraft bus, as seen in Figure 5. Mounted directly to the top surface of the bus is the barrel assembly, which is comprised of the barrel structure and the telescope lid. The barrel assembly encloses the payload, which includes the primary and secondary mirror assemblies, the primary support structure (PSS), the instrument bench with instruments and optics, the payload avionics, and the star trackers. The barrel has a scarfed baffle structure at the top. Along the height of the barrel are thin cylindrical ribs to suppress stray light. Mounted atop the PSS is the primary mirror assembly. The payload avionics are mounted to the underside of the PSS. The secondary mirror assembly is attached to the top of the inner barrel. The assembly is comprised of the secondary mirror, and the secondary support structure. The instrument bench is designed such that the optics and instruments are enclosed within the bench. Access holes have been designed into the top panel to enable installation and adjustment of the bench components. The payload is attached to the spacecraft bus at the PSS via a vibration isolation system to isolate the payload from bus disturbances. The payload contains two separate radiator panels. The instrument radiator panel attaches directly to the top instrument bench panel, while the payload avionics radiator mounts to the side of the PSS. Two star trackers, along with the star tracker electronics, attach to the barrel in between the instrument bench and the PSS. The star tracker electronics share a radiator with the payload electronics.

\subsection{Spacecraft}

The Exo-C spacecraft is designed to use significant Kepler heritage to meet the science requirements defined for the mission. With few exceptions, including structure, high-gain antenna (HGA), optics, and very reliable components, the spacecraft is designed to be fully redundant with all subsystems necessary to deliver the payload to orbit and support it through primary operations. The spacecraft utilizes a low-profile hexagonal box structure at the base of the coronagraph to minimize the total Flight Segment height and satisfy the fairing envelope constraints defined by intermediate class launch vehicles. The spacecraft meets all fairing volume constraints. The spacecraft utilizes a three-axis stabilized architecture, maintaining a fixed solar array pointed toward the Sun. This type of architecture minimizes jitter disturbances and shades the coronagraph telescope, helping to maintain payload thermal equilibrium. A body fixed Ka-band high-gain antenna (HGA) is used for high-rate data transmission with body-mounted X-band low-gain antennas (LGAs) for low-rate data transmission and commanding.

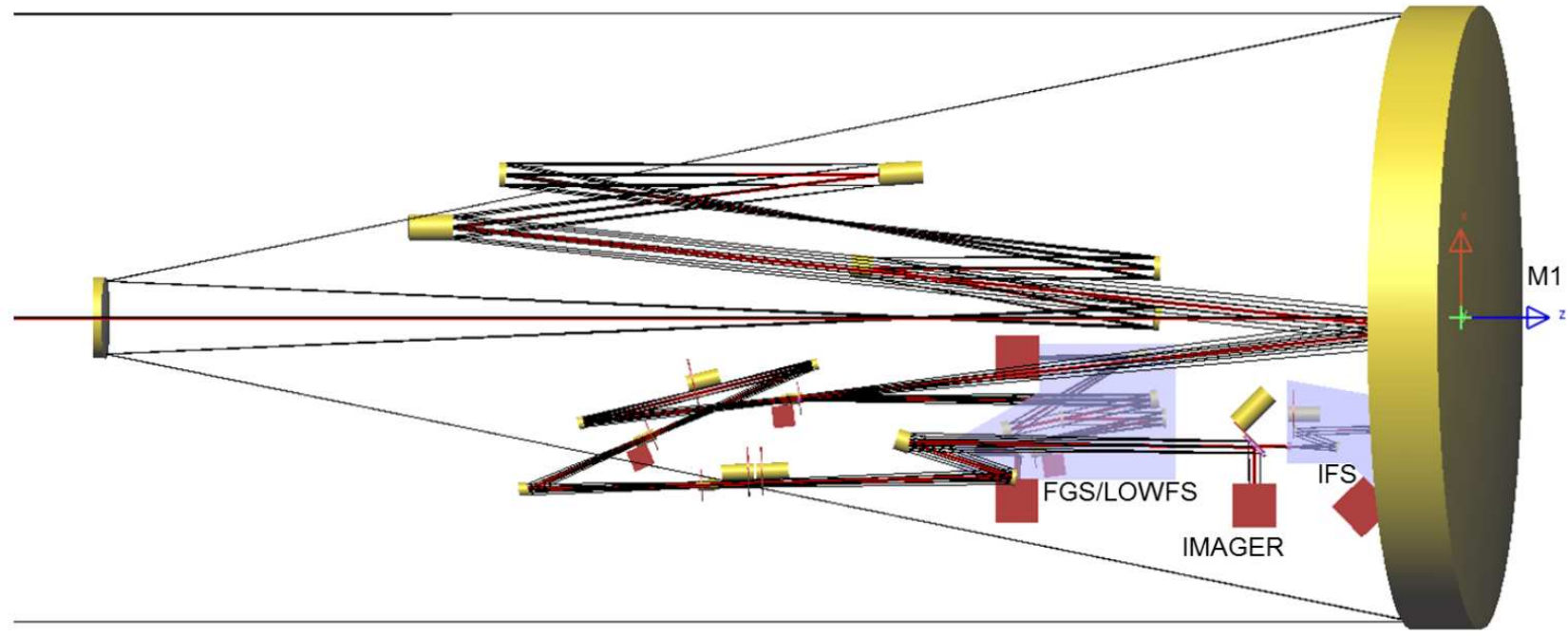

Figure 6. Baseline instrument bench layout at the Exo-C final study report. The lateral configuration along the anti-Sun side of the telescope includes two deformable mirrors, an FGS pointing sensor, a Low-Order WaveFront Sensor (LOWFS), FSM internal pointing mechanism, coronagraphic masks \& stops, spectral filters, and separate backend science camera heads for the imager and IFS. 


\subsection{Optical Configuration}

The optical portion of the payload (Figure 6) comprises the telescope and instrument assembly. The instrument assembly has two main subsections: the wavefront control optics and the coronagraph. Within these two subsections, there are subassemblies that support their indicated function. The control subsection contains a fine-guidance sensor (FGS) and a Low-Order WaveFront Sensor (LOWFS) used for pointing and wavefront error sensing and control, respectively. The final focal planes are the imager and the integral field spectrograph (IFS). The instrument assembly is located laterally with respect to the telescope axis, in a plane parallel to the telescope axis and offset to one side.

\subsection{Thermal}

The heliocentric, Earth-trailing orbit baselined for Exo-C is associated with extremely small planetary-based thermal loads, and the thermal design focuses on minimizing disturbances from varying incident solar loads. This is accomplished through the solar panel assembly, which also serves as a solar radiation shield. The barrel is covered with multi-layer insulation. The external layer minimizes solar absorption and resulting temperature increases. The barrel assembly serves as the secondary metering structure. It is actively temperature controlled to maintain its dimensional stability and to provide regulated constant temperature environment for the primary and secondary mirror surfaces, when subjected to varying solar incidence angles. Atop the barrel assembly is a scarfed solar shield to allow up to $45^{\circ}$ off-Sun pointing. Active heater control is also employed through radiative heating of the primary and secondary mirrors to reduce wavefront drift errors to acceptable levels within settling times of a few hours. The set-point temperature of the barrel is at room temperature to reject dissipations from payload electronics. The instrument bench runs at $<250 \mathrm{~K}$ and is used to maintain detector temperatures. The electronics dissipations are transported to the radiator via constant conductance heat pipes, while the detectors are sufficiently close to the radiator such that a solid-state link is used to transport waste heat.

\subsection{Low-order Wavefront Stability and Control}

Thermal changes on the primary mirror or in the primary/secondary despace will produce time-dependent loworder wavefront errors such as defocus, coma, and astigmatism. These errors can be crucial because they spread starlight near the inner working angle where many planet detections will take place. Exo-C has been designed for high thermal and mechanical stability, but changes in thermal state, optical wavefront, and contrast floor
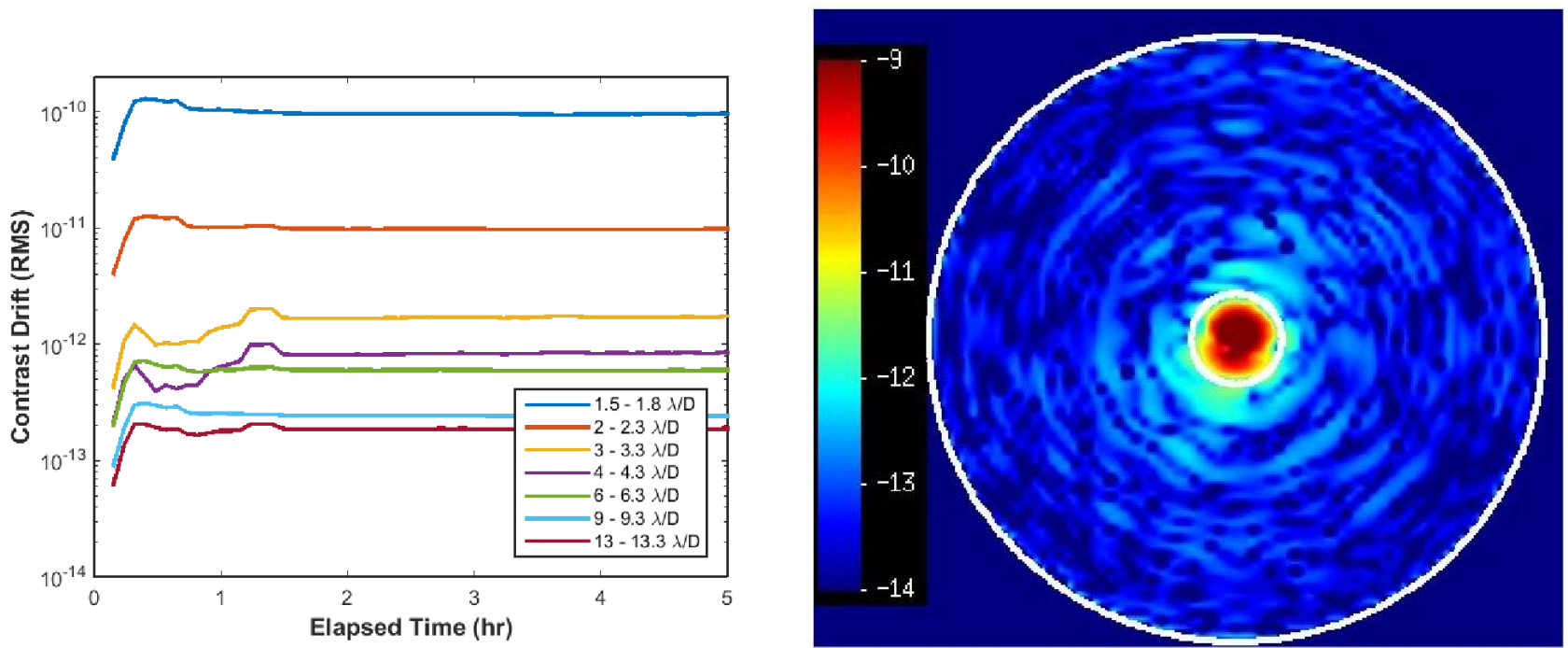

Figure 7 . Rolling the spacecraft from $-15^{\circ}$ to $+15^{\circ}$ induces very little wavefront or contrast drift because the thermal loads from this maneuver are symmetric on the flat panel sunshade. The panel at left shows the time evolution of the contrast drift through the roll maneuver, while the panel at at right shows the effect in the image plane. Comparable results are derived for a $30^{\circ}$ pitch maneuver 
in the coronagraph focal plane cannot be avoided when the telescope is maneuvered with respect to the input solar heating. Such maneuvers include slews between targets, and rolls about the telescope boresight (used to distinguish a planet from residual stellar speckles).

To quantitatively assess the low order wavefront stability of Exo-C, a Structural-Thermal-Optical model was set up. The thermal effects of the pitch and roll maneuvers, as a function of time, were modeled in the presence of the observatory's active thermal control system. Changes in the optical wavefronts were propagated through the coronagraph optical model to yield the changes in observed contrast in the image plane. The results (Figure 7) predict that the baseline Exo-C design will have excellent wavefront stability, allowing telescope maneuvers to be made routinely without the need to adjust either the low- or mid- frequency corrections made by the deformable mirrors. If achieved on orbit, this stability would obviate the need for a separate LOWFS and allow Exo-C to get by with FGS tip/tilt correction only. Further work is needed to confirm these very favorable results.

Despite the excellent optical wavefront stability predicted for Exo-C, the study team felt it was prudent to retain a low order wavefront control system as a contingency. The imaging camera and the IFS are not well suited for wavefront drift measurements because the suppression of the central star means very few photons are available on these cameras. For coronagraphs employing a focal-plane mask or other optical element such as a vector vortex, it is most effective to pick up light from the central star at an image plane upstream of the focal-plane mask where photons are plentiful. This is the approach taken for both the FGS and the LOWFS. Line of sight pointing drift is best handled by a dedicated FGS in a high bandwidth control loop driving a FSM in order to counteract spacecraft body pointing errors and jitter. A separate LOWFS samples slowly-varying WaveFront Error (WFE) terms and drives corrections to the DM settings during science exposures. Exo-C has baselined the Zernike LOWFS design. A dichroic layer placed on the front side of the coronagraphic mask would be used to reflect out-of-band light for use by the LOWFS and FGS.

\section{SCIENCE MISSION DESIGN}
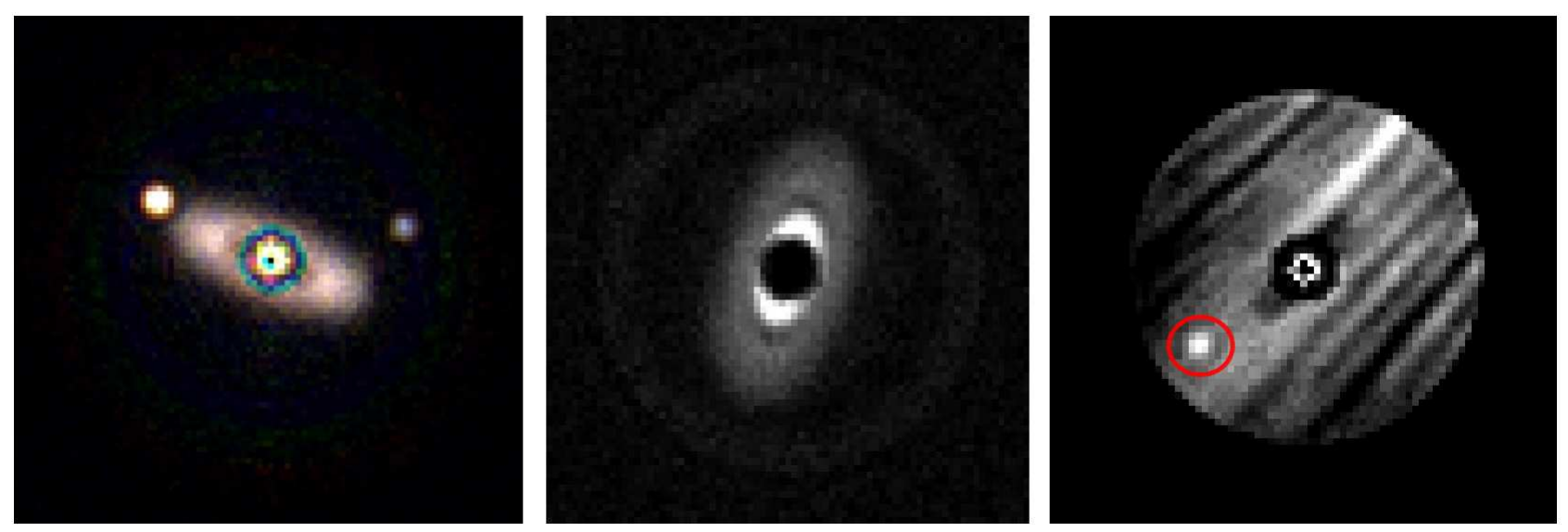

Figure 8. Simulated Exo-C imagery. Left: Hypothetical Jupiter and Saturn analogs around the nearby bright star Altair, along with a 1 zodi dust ring between 2-4 AU, as seen in 12 hour multicolor exposures. Center : 12 hour V band exposure showing hypothetical structure in the debris disk of HIP 85790, a star known to have 50 zodis of dust from WISE mission measurements. Right : 5 day $\mathrm{V}$ band exposure of an Earth analog in the habitable zone of $\alpha$ Centauri A. The diagonal streaks are due to scattered light from the companion star $\alpha$ Centauri B.

Based on our mission lifetime science observations budget, we expect to observe spectra of about 20 known and mission-discovered exoplanets, survey 15 nearby stars for super- Earths, and search 135 nearby stellar systems for the presence of giant planets. We also expect to image more than 200 circumstellar disks Integration times for the initial planet search observations are calculated using a detailed performance model for the coronagraph instrument and science detector, estimates of sky backgrounds, and assumed exoplanet properties such as planet radius, albedo and orbital illumination phase. The calculation uses contrast curves, mask profiles, system 
throughputs, and point-spread functions developed by the Exo-C Design Team. We make the conservative assumption that our survey of $135+15=150$ nearby stars discovers new exoplanets in only $10 \%$ of the targets. If spectra can be obtained for half of these 15 , then the total number of exoplanetary spectra returned by the mission would be around 20. Multicolor photometry can be obtained for 20 currently known exoplanets plus all 15 of the new discoveries. It should be noted that that some works project higher planet frequencies than $10 \%$, so Exo-Cs planet search could be substantially more fruitful than assumed here. Simulated images and spectra are shown in Figures 8 and 9.

\begin{tabular}{lcc}
\hline Science Program & $\begin{array}{c}\text { Number of } \\
\text { Science Targets }\end{array}$ & $\begin{array}{c}\text { Total Mission } \\
\text { Time (days) }\end{array}$ \\
\hline Exoplanet spectra & 20 & 215 \\
Exoplanet photometry \& astrometry & 35 & 166 \\
Super Earth discovery survey & 15 & 113 \\
Giant planet discovery survey & 135 & 323 \\
Habitable zone dust survey & 150 & 69 \\
Debris disk survey & 210 & 127 \\
Protoplanetary disk survey & 40 & 24 \\
\hline On-orbit checkout & & 60 \\
Mission Totals & 585 & 1097 \\
\hline
\end{tabular}

Table 1. Time allocations for a 3 year Exo-C mission. Roughly one year is devoted to planet searches; another year to photometry, astrometry, and spectroscopy of known and new planets; and the much of the remaining year to circumstellar disk imaging. Targets for exoplanet spectra are a subset of the photometry/astrometry targets.

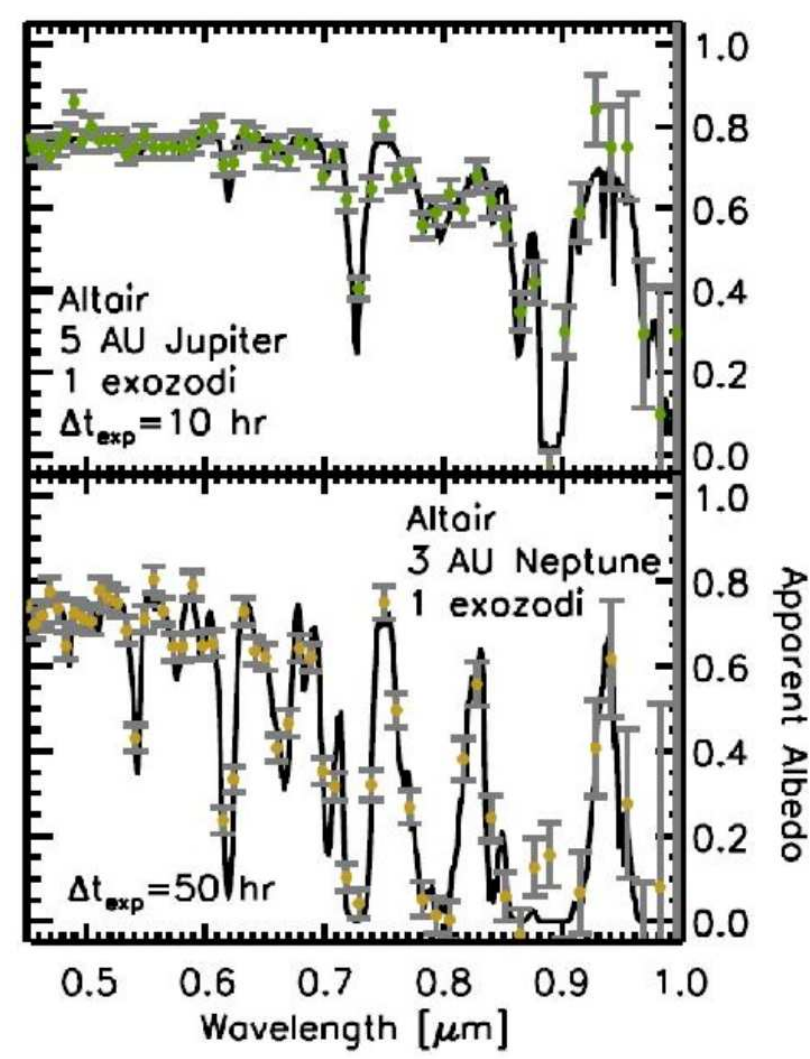

Figure 9. Simulated Exo-C spectra of hypothetical giant planets around the nearby bright star Altair.

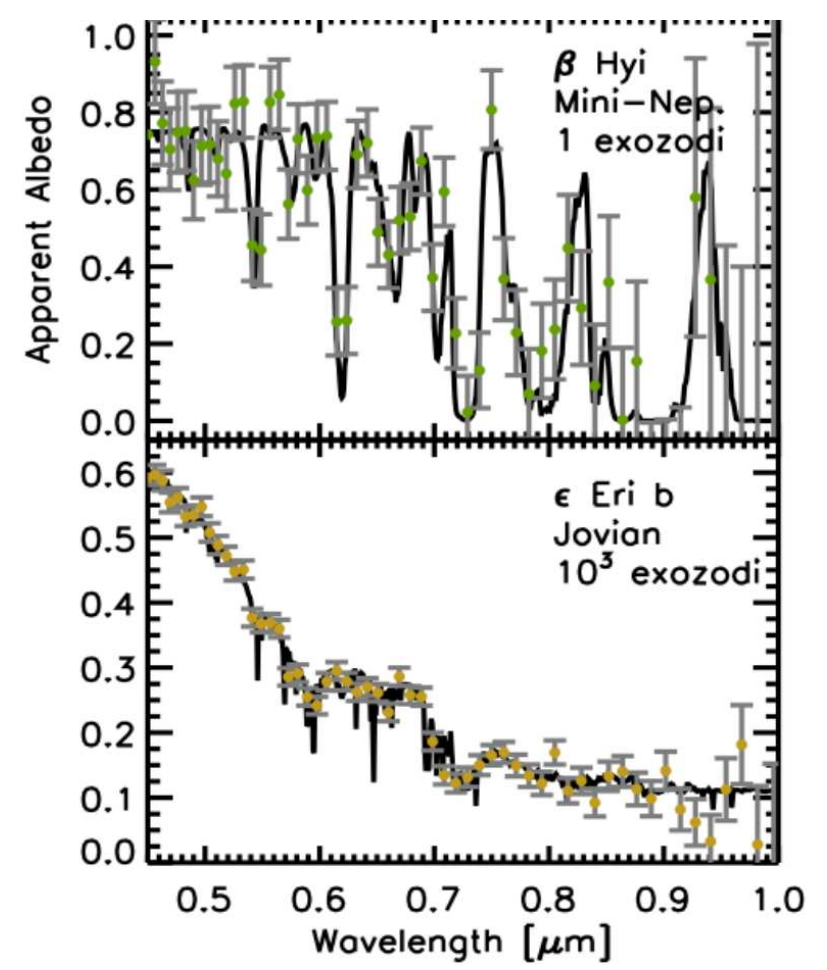

Figure 10. Simulated Exo-C spectra of a hypothetical miniNeptune planet around the nearby solar-type star $\beta$ Hydri; and of $\epsilon$ Eridani b, a Jupiter-sized planet and the closest confirmed exoplanet detected by the radial velocity technique. 


\section{TECHNICAL READYNESS}

Coronagraph Performance: Exo-C benefits from more than a decade of laboratory demonstrations of coronagraph performance with unobscured apertures in JPL's High Contrast Imaging Testbed (HCIT). These investments have matured the Hybrid Lyot coronagraph to a demonstrated contrast of $10^{-9}$ in $20 \%$ bandwidth at $3 \lambda / D$ inner working angle. For Exo-C, the required inner working angle is $2 \lambda / D$. The PIAA and Vector Vortex have demonstrated $10^{-8}$ contrast performance in $10 \%$ bandwidth at $2 \lambda / D$. All three coronagraphs therefore require additional developments and demonstrations in the next three years in order to meet Exo-C's requirements of $10^{-9}$ contrast in $20 \%$ bandwidth at $2 \lambda / D$ IWA. The AFTA/WFIRST mission study is currently investing in the Hybrid Lyot and PIAA coronagraph types, and can be expected to advance the state-of-the art in mask fabrication, apodizer fabrication, and flight qualification of deformable mirrors and low-noise detectors. A prototype IFS for high contrast is being funded separately by NASA HQ. All of these efforts will be beneficial for Exo-C. To be ready for a 2017 project start, Exo-C's major technical need is access to High Contrast Imaging Testbeds and funding to conduct further coronagraph demonstrations with unobscured pupils.

Telescope Stability: The greatest limitation to date for the laboratory coronagraph demonstrations cited above is that they have all been done in a static instrument. The real on-orbit instrument will have its performance degraded by pointing jitter that must be actively compensated for by a fine steering mirror. In addition, thermal or vibrational disturbances are likely to cause telescope focus and alignment to slowly drift, and these need to be compensated by wavefront control adjustments during long science exposures. Telescope pointing needs to be sensed and controlled to the 0.8 millarcsec level at high rates, as do the contrast-degrading effects of time-variable low-order telescope aberrations. There is a need for a dynamic coronagraph performance demonstration where the needed contrast is achieved in the presence of variable pointing and low-order aberrations. The AFTA/WFIRST mission study is setting up a new coronagraph testbed where the input stellar wavefront includes these temporal variations and where the a low-order wavefront sensor actively senses and corrects for them. This demonstration will be directly relevant to the needs of Exo-C. If successful, this would retire much of the risk of Exo-C meeting its performance requirements in a dynamic telescope environment.

Integral Field Spectrograph: Spectroscopic characterization of exoplanet atmospheres is one of the primary science goals of the mission and the integral field spectrograph (IFS) has been chosen as the most promising technology for efficient capture of the spectra. The IFS is a proven technology utilized widely on large groundbased telescopes, but the IFS has yet to be demonstrated in a flight environment. The first lenslet-based IFS was a visible-light instrument at the Canada France Hawaii Telescope (Bacon et al. 1995), and later it was proven to also be viable in the infrared with the OH-Suppressing InfraRed Imaging Spectrograph (OSIRIS) IFS at Keck (Larkin et al. 2006). Now, all of the next-generation, ground-based, high-contrast imaging systems include lenslet-based IFSs as their science cameras, therefore justifying this instrument concept at Technology Readyness Level 4. The only nontraditional optic in a lenslet-based IFS is the lenslet array itself. Lenslet arrays have been used to conduct science at low contrast on ground-based telescopes for the past 18 years. However, lenslet arrays need to demonstrate that they can meet the $10^{4}$ spectral crosstalk requirements of a space-based high contrast imager. A prototype IFS designed to demonstrate the needed performance (PISCES; McElwain et al. 2013), will be deployed to the HCIT in early 2016.

\section{ISSUE FOR FURTHER STUDY}

The Kepler photometer is exquisitely sensitive to spacecraft disturbances, as these create systematic errors that must be filtered out of the stellar lightcurves to identify plantary transits. The Kepler lightcurves therefore contain valuable information on telescope stability in an Earth-trailing orbit, and should be analyzed more fully. Such an analysis could reveal 1) how well Kepler mission designers did in isolating the science instrument from disturbances; 2) a quantitative measurement of the telescope response to spacecraft system changes, useful for validation of Structural/Thermal/OPtical models of very low amplitude disturbances; and 3) additional disturbance sources that might be mitigated by engineering improvements. NASA, the Kepler data team, and the Ball Aerospace engineering team should pursue this work in order to fully learn the spacecraft stability lessons Kepler can teach us.. 


\section{SUMMARY AND CONCLUSIONS}

Design work during 2014 focused on reducing the cost and complexity of the system and improving wavefront stability. The telescope was downsized from a $1.5 \mathrm{~m}$ to $1.4 \mathrm{~m}$ aperture, the solar panels were expanded into a flat sunshield for the entire telescope (enabling the interim design of two concentric barrels to be downsized to a single barrel), the pointing requirements were relaxed based on coronagraph performance simulations, and the design of the instrument optical bench was refined. The final design remains very similar to that of Kepler: the same telescope aperture, orbit, mission lifetime, spacecraft and launch vehicle requirements. Internal study cost estimates are just below the Probe mission cost cap specified by NASA HQ. Independent estimates are only slightly higher. The Exo-C Science and Engineering teams thus believe that the study has succeeded in its charge to produce a compelling science mission at or below the imposed cost cap of $\$ 1 \mathrm{~B}$.

After the start of the Exo-C study, NASA committed to adding a coronagraph instrument to the AFTA/WFIRST mission. While AFTA features a $2.4 \mathrm{~m}$ telescope, its obscured pupil strongly limits coronagraph performance. Preliminary comparisons indicate that Exo-C, with its much smaller but optimal telescope, would achieve comparable or better exoplanet imaging performance than the AFTA coronagraph. However AFTA/WFIRST will serve a larger user community, and the addition of the coronagraph to the mission will be much less expensive than flying a separate Exo-C mission. Thus under the current Agency plan, AFTA/WFIRST will go forward and Exo-C will not proceed beyond the study phase. Even so, our study team is hopeful that many of the optimal design features we identified (lateral instrument bench, precise thermal control of the telescope, flat panel sunshade, stiff spacecraft design, etc.) will find their way into a future exoplanet flagship mission.

The contents of this paper have been distilled from the March 2015 Exo-C Final Study Report, available in full at http://exep.jpl.nasa.gov/stdt. This material has been approved for external release, JPL CL\#15-0026, We thank the staff of the Exoplanet Exploration Program Office at JPL for their assistance in many aspects of this study. This work has been supported by funding from the Astrophysics Division, Science Mission Directorate, NASA Headquarters.

\section{REFERENCES}

Bacon, R.Y. et al. 1995, MNRAS, 326, 23

Cahoy, K., Marley, M., and Fortney, J. 2010, Ap.J. 724189

Carlotti, A., Kasdin, N. J., Vanderbei, R. J., \& Delorme, J.-R. 2012, Proc. SPIE, 8442

Clampin, M., \& Lyon, R. 2010, Pathways Towards Habitable Planets, 430, 383

Guyon, O., Shaklan, S., Levine, M., et al. 2010, Proc. SPIE, 7731,

Guyon, O., \& Martinache, F. 2012, Proc. SPIE, 8442

Howard, A. and Fulton, B.J. 2015

http://exep.jpl.nasa.gov/reportsAndDocuments/completenessStudy/rv_limits_report.pdf

Karkoschka, E. 1994, Icarus 111174

Larkin, J. et al. 2006, New Astronomy Reviews 50, 362

Lawson, P.R. et al. 2013, Proc. SPIE, 8864

Lyon, R. G., Clampin, M., Petrone, P., et al. 2012, Proc. SPIE, 8442

Mawet, D., Serabyn, E., Moody, D., et al. 2011, Proc. SPIE, 8151

McElwain, M. et al. 2013, Proc. SPIE, 8864

Trauger, J., Stapelfeldt, K., Traub, W., et al. 2010, Proc. SPIE, 7731

Trauger, J., Moody, D., Gordon, B., Krist, J., \& Mawet, D. 2012, Proc. SPIE, 8442 